\title{
A study of spontaneous echo contrast in patients with rheumatic mitral stenosis and normal sinus rhythm: an Indian perspective
}

\author{
R R Kasliwal, S Mittal, A Kanojia, R P Singh, O Prakash, M L Bhatia, N Trehan
}

\begin{abstract}
Objective-To study the incidence of spontaneous echo contrast in left atrium of Indian patients with rheumatic mitral stenosis in normal sinus rhythm and to define its relations.

Subjects-Transthoracic and multiplane transoesophageal echocardiographic studies were performed in 89 consecutive patients with rheumatic mitral stenosis who were in normal sinus rhythm.

Results-Spontaneous echo contrast in the left atrium was seen in $57.3 \%$ of patients on multiplane transoesophageal echocardiography and in only $5.6 \%$ on transthoracic echocardiography. The mean mitral valve area was 1.07 (SD $0.33) \mathrm{cm}^{2}$ and $1.32(0.45) \mathrm{cm}^{2}(P=0.004)$, mean left atrial size was $4.27(0.67) \mathrm{cm}$ and $3.91(0.5) \mathrm{cm}(P=0.029)$, mean diastolic pressure gradient was $12.64(5 \cdot 69)$ $\mathrm{mm} \mathrm{Hg}$ and 10 (5.5) $\mathrm{mm} \mathrm{Hg}(P=0.049)$, and absence of mitral regurgitation was seen in $45 \%$ and $23 \%$ of patients respectively $(P=0 \cdot 1)$. Among patients with spontaneous echo contrast, $31 \%$ had either left atrial/appendage thrombus or a history of embolism, $v 0 \%$ in patients without spontaneous echo contrast $(P<$ 0.0001).

Conclusions-There is a high incidence of spontaneous echo contrast in the left atrium in Indian patients with rheumatic mitral stenosis in normal sinus rhythm on multiplane transoesophageal echocardiography. These patients are likely to embolise or form thrombi in the left atrium. The presence of spontaneous echo contrast is also associated with significantly smaller mitral valve area, larger left atrium, and higher mean diastolic mitral pressure gradient.
\end{abstract}

(Br Heart f 1995;74:296-299)

Institute and Research Centre, New Delhi, India

R R Kasliwa

$S$ Mittal

A Kanojia

R P Singh

O Prakash

M L Bhatia

N Trehan

Correspondence to:

Dr R R Kasliwal, Senio

Consultant Cardiology,

Escorts Heart Institute and

Research Centre, Okhla

Road, New Delhi-110025, India.

Accepted for publication 1 March 1995
Quantification of mitral regurgitation, localisation of left atrial and left atrial appendage thrombi, and delineation of sub- valvular pathology can be done more accurately by multiplane transoesophageal echocardiography than by transthoracic echocardiography. ${ }^{2-4}$ Another important finding frequently seen in patients with rheumatic mitral stenosis on multiplane transoesophageal echocardiography is spontaneous echo contrast, that is, dynamic clouds of echoes curling slowly in a circular or spiral manner within the left atrial cavity. Studies show that spontaneous echo contrast is a predictor of thromboembolism in patients with mitral stenosis. ${ }^{356} \mathrm{We}$ know that patients with atrial fibrillation have a high incidence of spontaneous echo contrast but its incidence in patients in normal sinus rhythm is in no way negligible. The role of spontaneous echo contrast in patients with normal sinus rhythm is still not clear.

Thus we undertook this study to elucidate the incidence of spontaneous echo contrast in patients with rheumatic mitral stenosis in normal sinus rhythm and to define its relations with left atrial size, degree of mitral regurgitation, mean diastolic mitral pressure gradient, mitral valve area, presence of thrombus in left atrium or its appendage, and history of embolism.

\section{Methods}

PATIENTS

We studied 89 consecutive patients with rheumatic mitral stenosis in normal sinus rhythm presenting to the outpatients clinics of our hospital from January 1993 to June 1994 Patients who were non-cooperative, had active gastrointestinal bleeding, or had oesophageal obstruction were excluded from the study.

\section{PROCEDURES}

All patients were subjected to detailed transthoracic echocardiography using HewlettPackard Sonos 1500. Transoesophageal echo was performed in a specialised suite using a Hewlett-Packard Sonos 1500 with an omniplane 64-element phased array transducer probe having a dual frequency feature $(5 / 3.5$ $\mathrm{MHz}$ ) pulsed, continuous wave, and colour Doppler facilities.

All patients were subjected to overnight or at least four hours of fasting. After obtaining informed consent, the patient's throat was sprayed with $10 \%$ lignocaine solution to anaesthetise the oropharynx. No other preprocedural medication was given.

The patients lay in the left lateral position 
with the head flexed. A mouth guard was then introduced and patients were asked to hold their lips around it. The transoesophageal probe was passed through the central hole of the mouth piece to reach the oropharynx. At this point, the probe was slightly anteflexed and the patients were asked to swallow. This smoothened the entry into the oesophagus. The probe was brought to neutral position and glided gently till it was in position behind the left atrium. After the probe had been introduced, an effort was made to acquire goal oriented images. Subsequently, and in a well defined sequence, transgastric images were obtained (of both the left and the right heart), followed by imaging from the gastrooesophageal junction. The probe was then withdrawn a little and images were acquired from low and mid-oesophagus level, and finally from the upper oesophagus. At each level the tip of the transducer was moved from $0^{\circ}$ to $180^{\circ}$ and back to obtain a complete study of the heart and great vessels.

Left atrial diameter was measured according to standard $M$ mode criteria on transthoracic echo. ${ }^{7}$ Mitral valve area was determined by the Doppler study, using the pressure half time method on transoesophageal echocardiography. ${ }^{8}$ Mitral stenosis was defined as severe when mitral valve area was less than $1 \mathrm{~cm}^{2}$, moderate if it was $1-1.5$ $\mathrm{cm}^{2}$, and mild if it was over $1.5 \mathrm{~cm}^{2}$. Left atrial spontaneous echo contrast was defined as a dynamic cloud of echoes swirling slowly within the left atrial cavity. When it was present, the gain was systematically decreased in a stepwise fashion to exclude noise artefacts caused by excessive gain, and an optimum image gain was obtained. Left atrial thrombus was diagnosed as a dense well circumscribed echo mass acoustically distinct from the underlying endocardium. In patients where mitral insufficiency was present, the severity of mitral regurgitation was judged by measur-

Table 1 Distribution of cases according to severity of mitral stenosis

\begin{tabular}{llll}
\hline & Mitral valve area & \\
\cline { 2 - 4 } & $\geqslant 1.6 \mathrm{~cm}^{2}$ & $1-1.5 \mathrm{~cm}^{2}$ & $\geqslant 0.9 \mathrm{~cm}^{2}$ \\
\hline Total number & $13[14 \cdot 6 \%]$ & $51[57.3 \%]$ & $25[28 \cdot 1 \%]$ \\
Spontaneous echo contrast & $4[30 \cdot 8 \%]$ & $29 \star[56.9 \%]$ & $18 \dagger[72 \%]$ \\
Pressure gradient (mm Hg), mean (SD) & $7.38(4.68)$ & $10.47(5.26)$ & $15.79(4.64)^{\star}$ \\
\hline${ }^{\star}<00.05 ; \mathrm{P}<0.001$. & &
\end{tabular}

Table 2 Relation of spontaneous echo contrast (SEC) with various echocardiographic variables. Values are means (SD)

\begin{tabular}{lllr}
\hline Variables & SEC present & SEC absent & P Value \\
\hline MVA $\left(\mathrm{cm}^{2}\right)$ & $1.07(0.33)$ & $1.32(0.45)$ & 0.004 \\
Absence of MR (\%) & 45 & 23 & 0.1 \\
LA size (cm) & $4.27(0.67)$ & $3.91(0.58)$ & 0.029 \\
Mean PG (mm Hg) & $12.64(5.69)$ & $10.01(5.51)$ & 0.049 \\
History of thrombus in & & & $<0.0001$ \\
LALAA & $\mathrm{n}=9$ & $\mathrm{n}=0$ & $<0.0001$ \\
History of embolism & $37.9(7.3)$ & $67.2(8.3)$ & $<0.0001$ \\
Emptying velocity (cm/s) & $28.6(7.5)$ & $57.2(10.0)$ & $<0.0001$ \\
Filling velocity $(\mathrm{cm} / \mathrm{s})$ & & &
\end{tabular}

MVA, mitral valve area; LA, left atrium; LAA, left atrial appendage; MR, mitral regurgitation; PG, pressure gradient. ing the area of the colour flow jet and calculating it as a percentage of the area of left atrium. ${ }^{9}$

\section{STATISTICAL ANALYSIS}

We used the $\chi^{2}$ test with Yates's correction to compare difference in groups. Student's $t$ test and the $\mathrm{F}$ test were applied to test the significance of differences between individual variables where appropriate. Values are given as mean (SD). In all statistical analyses, $\mathrm{P}<$ 0.05 was considered significant.

\section{Results}

Out of the 121 patients with rheumatic mitral stenosis, 89 were in normal sinus rhythm and these formed our study sample. Of these 31 $(34 \cdot 8 \%)$ were males and $58(65 \cdot 2 \%)$ were females. The mean age of our patients was $36 \cdot 4(10 \cdot 6)$ years, range $19-70$ years.

Mitral regurgitation was present in 56 patients $(63 \%)$. Among patients with spontaneous echo contrast, $55 \%$ had mitral regurgitation, while this was present in $77 \%$ of those without spontaneous echo contrast.

Mean diastolic pressure gradient was found to be $11.51(5.54) \mathrm{mm} \mathrm{Hg}$ and it increased in accordance to the severity of mitral stenosis, as shown in table 1. Spontaneous echo contrast was seen within the atrial cavity in $\mathbf{5 1}$ patients $(57 \cdot 3 \%)$ on transoesophageal echo. However only five $(5.6 \%)$ revealed spontaneous echo contrast on transthoracic echocardiography. In the remaining 32 patients not included in the study sample, who had mitral stenosis and were in atrial fibrillation, we found a $\mathbf{7 8 \%}$ incidence of spontaneous echo contrast.

Mean mitral valve area in our patients was $1.18(0.5) \mathrm{cm}^{2}$ and the mean left atrial size was $4.11(0.68) \mathrm{cm}$. The mitral valve area was smaller $(P<0.005)$, the left atrial size was larger $(P<0.05)$, and mean diastolic pressure gradient was higher $(P<0.05)$ in patients with spontaneous echo contrast than in those without. Absence of mitral regurgitation was more often found in patients with spontaneous echo contrast than in those without; however, the difference was not significant $(P=0 \cdot 1)$, as shown in table 2 .

Three patients $(3.4 \%)$ showed presence of thrombus in left atrium or appendage on transthoracic echo, whereas on transoesophageal echo nine patients $(10 \cdot 1 \%)$ were found to have left atrial or appendage thrombi. All patients with left atrial or appendage thrombi had spontaneous echo contrast in left atrium (table 2). Nine patients $(10.1 \%)$ had a history of embolism in the form of transient ischaemic attacks, cerebrovascular accidents, and peripheral embolism. All of these patients had spontaneous echo contrast in the left atrium. The presence of left atrial or appendage thrombus, however, did not correlate with a history of embolism. Overall, $31 \%$ of patients with spontaneous echo contrast had either left atrial or appendage thrombus on transoesophageal echo or a history of embolism, whereas none 
of those without spontaneous echo contrast had either $(P<0.0001)$ (table 2$)$.

We estimated the flow velocities in and out of the left atrial appendage by pulsed wave Doppler in our patients. The mean emptying velocity was $37.9(7.3) \mathrm{cm} / \mathrm{s}$ in patients with spontaneous echo contrast and was significantly less than in those without spontaneous echo contrast. Similarly filling velocities were significantly lower in patients with spontaneous echo contrast compared to those without spontaneous echo contrast (table 2 ).

\section{Discussion}

Our study is the only one of its kind which has been done exclusively in patients with rheumatic mitral stenosis in normal sinus rhythm. Iliceto et al ${ }^{10}$ performed a transthoracic echo study on 10 patients with mitral stenosis and spontaneous echo contrast and all were in atrial fibrillation. Similarly Beppu et $a l^{5}$ performed a transthoracic echocardiographic study on 116 consecutive patients, of whom 49 had mitral valve disease. Kranidis et $a l^{3}$ studied 101 consecutive patients with mitral valve disease of whom only 57 had rheumatic valve disease and the sample included patients in atrial fibrillation and in normal sinus rhythm.

In studies done by Black et $a^{6}{ }^{6}$ and Castello et $\mathrm{al}^{4}$ it was not clearly defined how many patients with mitral stenosis were in atrial fibrillation or normal sinus rhythm. However, Daniel et $a l^{11}$ in a study of 52 patients with mitral stenosis divided his patients into those with atrial fibrillation and those in normal sinus rhythm.

\section{PATIENT BACKGROUND}

The mean age of patients in our study was 36.4 years, which is less than in earlier studies. This may be attributable to the fact that rheumatic heart disease in India has a relatively high incidence in the younger population. In the present study a female preponderance was observed, which is in accordance with previous studies. ${ }^{35}$

INCIDENCE OF SPONTANEOUS ECHO CONTRAST In our study spontaneous echo contrast was detected in $57.3 \%$ patients on multiplane transoesophageal echo, whereas only $5.6 \%$ were picked up on transthoracic echo. The incidence of spontaneous echo contrast was significantly higher in patients with atrial fibrillation. Daniel et $a l^{11}$ found spontaneous echo contrast in $43.1 \%$ of patients with mitral stenosis in normal sinus rhythm. However the sample size in their study was smaller than ours (only 16 patients). Other studies ${ }^{36}$ also report a high incidence $(55-60 \%)$ in their studies. However the study samples included patients in atrial fibrillation as well.

\section{SPONTANEOUS ECFTO COMTRAST AND TTS RELATSONS}

In our study the presence of spontaneous echo contrast was inversely related to mitral valve area. Similar results have been reported by others ${ }^{356}$; however, they also included patients with mitral stenosis who were in atrial fibrillation.

In the present study the incidence of spontaneous echo contrast decreased with increasing grades of mitral regurgitation but the trend was not significant. Black et $a l^{6}$ noted a significant relation between the presence of mitral regurgitation and spontaneous echo contrast; however, their study population included patients with disorders other than rheumatic mitral valve disease. Kranidis et $a l^{3}$ noted similar findings; on the other hand Castello et $a l^{4}$ found that mitral regurgitation was directly related to the presence of spontaneous echo contrast in the left atrium. The discrepancy may be attributable to the difference in the study population. Significant mitral regurgitation may prevent the development of left atrial spontaneous echo contrast by provoking a stirring effect in the left atrium.

A significant number of patients $(10 \cdot 1 \%)$ in our study had left atrial or appendage thrombi on transoesophageal echo. The incidence of left atrial or appendage thrombi in those who had spontaneous echo contrast was even higher $(17 \cdot 7 \%)$. Daniel et $a l^{11}$ reported thrombi in $15.3 \%$ of their patients with normal sinus rhythm and in $22.3 \%$ of those with spontaneous echo contrast (including patients in normal sinus rhythm as well as with atrial fibrillation). The results are comparable to ours.

A history of embolism was found in $10.1 \%$ of our patients overall, and in $17.7 \%$ of patients with spontaneous echo contrast. According to Daniel et al, ${ }^{11}$ a history of embolism in patients with spontaneous echo contrast is present in $37 \%$, but this includes patients with atrial fibrillation as well.

Overall, $31 \%$ of patients with spontaneous echo contrast had either thrombus in the left atrium or appendage, or a history of embolism in the past, whereas none of the patients without spontaneous echo contrast had either of these features. This cannot be ignored.

Mean left atrial size and mean diastolic mitral pressure gradient in patients with spontaneous echo contrast were significantly higher than those without spontaneous echo contrast. This observation is similar to that reported by others. ${ }^{3611}$

We noted that spontaneous echo contrast was associated with significantly lower filling and emptying velocities in the left atrial appendage than in patients without spontaneous echo contrast. Our findings are similar to those of Kranidis et $a l,{ }^{3}$ although they included patients with atrial fibrillation in their study. Pollick and Taylor ${ }^{12}$ also reported similar findings.

In our study the factors that favoured the development of spontaneous echo contrast were a larger left atrium, a smaller mitral valve area, a higher mean diastolic pressure gradient, and lower filling and emptying velocities in the left muinl appundape. All these factors result in stasis in the left atrial appendage.

Although the pathophysiology of spontaneous echo contrast is unknown, it has 
been postulated that rouleaux formation of erythrocytes occurring during low flow conditions may be responsible. ${ }^{13}$ Turbulence could also enhance echogenicity by disrupting aggregated and non-aggregated erythrocyte orientation and thus causing ultrasonic back scatter. ${ }^{14}$ Spontaneous echo contrast in the rabbit has been shown to be caused by circulating platelet aggregates that are filtered incompletely by the lungs. ${ }^{15}$ Echogenicity of whole blood is shear dependent and flow dependent. Spontaneous echo contrast in clinical states such as a dilated left atrium in mitral stenosis may be generated by the high velocity incoming blood meeting a stagnant blood pool. ${ }^{16}$ At times the echoes may be more dense than spontaneous echo contrast within the cavity of the left atrium but less dense than the solid echogenic thrombus. This intermediate state may be a more gelatinous form of early thrombus formation.

\section{CLINICAL IMPLICATIONS}

It is clear from this study that Indian patients with rheumatic mitral stenosis have a significant incidence of left atrial or appendage thrombi and thromboembolic episodes, even though they may be in normal sinus rhythm. The presence of spontaneous echo contrast in these patients is associated with a significant risk of thromboembolic episodes. A close relationship between left atrial or appendage thrombi and spontaneous echo contrast may be due to the fact that the factors responsible for the generation of either of these are similar. The importance of spontaneous echo contrast in patients with rheumatic mitral stenosis in normal sinus rhythm is no less than in those who are in atrial fibrillation, as has been reported by others. ${ }^{3611}$

With such a high potential for thrombus formation it is tempting to think that in patients with rheumatic mitral stenosis who are in normal sinus rhythm and who show spontaneous echo contrast in the left atrium on multiplane transoesophageal echo, pharmacological (antiplatelet and anticoagulant) or interventional measures should be undertaken instead of waiting for the formation of thrombus or embolism to occur. Therapeutic studies have been done to investigate the resolution of spontaneous echo contrast.
According to Mahony, ${ }^{17}$ resolution occurs after five days of therapy with trifluperazine, but Hoffman ${ }^{18}$ found persistence of spontaneous echo contrast despite therapy with trifluperazine, and its density remained unchanged. The issue of the resolution of spontaneous echo contrast following anticoagulant treatment remains controversial and requires further study.

1 Mitchell MM, Sutherland GR, Gussenhoven EJ, Taams MA, Roelandt J. Transesophageal echocardiography. f Am Soc Echocardiogr 1988;1:362-77.

2 Cooper JW, Nanda NC, Philpot EF, Fan P. Evaluation of valvular regurgitation by color Doppler. f $\mathrm{Am} S o c$ Echocardiogr 1989;2:56-66.

3 Kranidis A, Koulouris S, Anthopoulos L. Clinical implications of left atrial spontaneous echo contrast in mitral valve disease. 7 Heart Valve Dis 1993;2:267-72.

4 Castello R, Pearson AC, Labovitz AJ. Prevalence and clinical implications of atrial spontaneous contrast in patients undergoing transoesophageal echocardiography. Am $\mathcal{F}$ Cardiol 1990;65:1149-53.

5 Beppu S, Nimura Y, Sakakibara H. Smoke like echo in left atrial cavity in mitral valve disease: its features and significance. $\Im$ Am Coll Cardiol 1985;6:744-9.

6 Black IW, Hopkins AD, Lee LC. Left atrial spontaneou echo contrast: a clinical and echocardiographic analysis. f Am Coll Cardiol 1991;18:398-404.

7 Engle SJ, Disessa TG, Perloff JK, Isabel-Jones J, Leighton $\mathrm{J}$, Gross $\mathrm{K}$, et al. Mitral valve $\mathrm{E}$ point to ventricular septal separation in infants and children. Am $\mathcal{f}$ Cardio 1983;52:1084-7.

8 Hatle L, Brubakk A, Tromsolal A, Angelsen B. Non invasive assessment of pressure drop in mitral stenosis by Doppler ultrasound. Br Heart $₹$ 1978;40:131-40.

9 De Masia AN, Smith MD Quantitation of Doppler color flow recordings: an oxymoron? $¥ \mathrm{Am}$ Coll Cardiol 1992; 20:439-40.

10 Iliceto $S$, Antonelli G, Sorino M. Dynamic intra cavitary left atrial echoes in mitral stenosis. Am $\mathcal{F}$ Cardiol 1985; 55:603-6.

11 Daniel WG, Nellesen U, Schroder E. Left atrial spontaneous echo contrast in mitral valve disease: an indicato for an increased thromboembolic risk. $\mathcal{F} \mathrm{Am}$ Coll Cardiol 1988;11:1204-11.

12 Pollick C, Taylor D. Assessment of left atrial appendage function by transoesophageal echocardiography. Implications for the development of thrombosis. Circulation 1991;84:223-31.

13 Siegel B, Coelho JO, Spigos DG. Ultrasonography of blood stasis and coagulation. Invest Radiol 1981;16: $71-6$.

14 Chandrasekaran $\mathrm{K}$, Ross J, Covolesky VA. Two dimensional echocardiographic visualization of turbulent sional echocardiographic visualization of turbulent Heart $¥ 1989 ; 118: 625-7$.

15 Mahony C, Ayappa IA, Ferguson J. Spontaneous contrast in the rabbit. Circulation (suppl II) 1991;84:692 (abstr).

16 Merino A, Hauptman PJ, Badimon L. Echogenic smoke is shear rate dependent. Circulation (suppl II) 1991;84:692 (abstr)

17 Mahony C, Kevil L, Michael R. Resolution of spontaneous contrast with platelet disaggregatory therapy (trifluoperazine). Am F Cardiol 1989;63:1009-10.

18 Hoffman R, Lambertz H, Kreis A. Failure of trifluoperazine to resolve spontaneous echo contrast evaluated by transoesophageal echocardiography. Am 7 Cardiol 1990 66:648-50. 